\title{
Investigation on inkjet printing for electromagnetic compatibility application
}

\author{
Melinda Hartwig ${ }^{1}$, Maxim Polomoshnov ${ }^{1}$, Ralf Zichner ${ }^{2}$, Reinhard R. Baumann ${ }^{1,2}$ \\ 1) Department of Digital Printing and Imaging Technology, melinda.hartwig@mb.tu-chemnitz.de, \\ maxim.polomoshnov@mb.tu-chemnitz.de, reinhard.baumann@mb.tu-chemnitz.de, Technische \\ Universität Chemnitz, Reichenhainer Straße 70, 09126 Chemnitz, Germany \\ 2) Department of Printed Functionalities, ralf.zichner@enas.fraunhofer.de, Fraunhofer Institute for \\ Electronic Nano Systems (ENAS), Technologie-Campus 3, 09126 Chemnitz, Germany
}

\section{Keywords}

Inkjet printing, high accuracy, electromagnetic compatibility (EMC), frequency-dependent attenuation of electromagnetic waves

\begin{abstract}
The research focused on the development of inkjet-printed silver grids on flexible films to (I) attenuate electromagnetic waves at $2.45 \mathrm{GHz}$ locally by applying them directly on Wi-Fi or Bluetooth transmitter or to (II) protect electromagnetic compatibility (EMC) sensitive devices close to electromagnetic transmitters. The inkjet printing technology leads to resource, time as well as cost efficient manufacturing and simplifies the adjustment of the pattern design regarding different applications. The research contains a fundamental analysis of the behavior of printed silver patterns on flexible polymer substrates regarding line widths, layer morphology and electrical performance. On that basis, the grid pattern for a certain frequency range is simulated. The report shows first simulation results on basis of the given material parameters. The results are the fundamentals for further research on realization of the simulated grid patterns by inkjet printing technology.
\end{abstract}

\section{Introduction}

The inkjet printing technology is an additive and therefore economic manufacturing process, since the material is applied only on areas where it is needed. Electronic devices like resistors, capacitors [1], antennas [2] and further functional thin film patterns can be realized with small amounts of material. Due to the fact that it is a digital and contactless printing technology the layout can easily be adjusted for different purposes on the one hand and on the other hand various substrates especially flexible ones can be used and roll-to-roll processed. This circumstances lead to the possibility to combine the printing process with various manufacturing methods e.g. to produce smart lightweight composites [3].

In this research inkjet-printed silver grids are investigated with regard to accurate line geometries which are given by simulations. The challenge is to realize printed patterns which deviate from the simulated ones as less as possible to realize a defined and reproducible attenuation or shielding especially at high frequency ranges [4]. Two possible applications are considered. On the one hand the attenuation of electromagnetic radiation of commercial devices like Wi-Fi and Bluetooth applications (e.g. in automobiles) in a specified frequency range to protect these wireless networks from unwanted external access as well as disturbances. On the other hand to ensure a protection of EMC sensitive devices close to electromagnetic transmitters with high output power (e.g. radar, magnetic resonance tomography). Figure 1 demonstrates schematically the manufacturing process and the two potential applications of the printed silver grids.

State of the art demonstrates that there is a need of electromagnetic shielding especially from healthcare and data security point of view $[5,6]$. To replace currently used complex manufacturing processes e.g. conventional printed circuit board technologies like chemical etching [7, 8] and spray coating [9] or thick 
screen printed layers [10], the inkjet printing technology has great potential to fulfill the requirements of minimal material input by narrow patterns and small layer thickness with simultaneous performance accuracy.

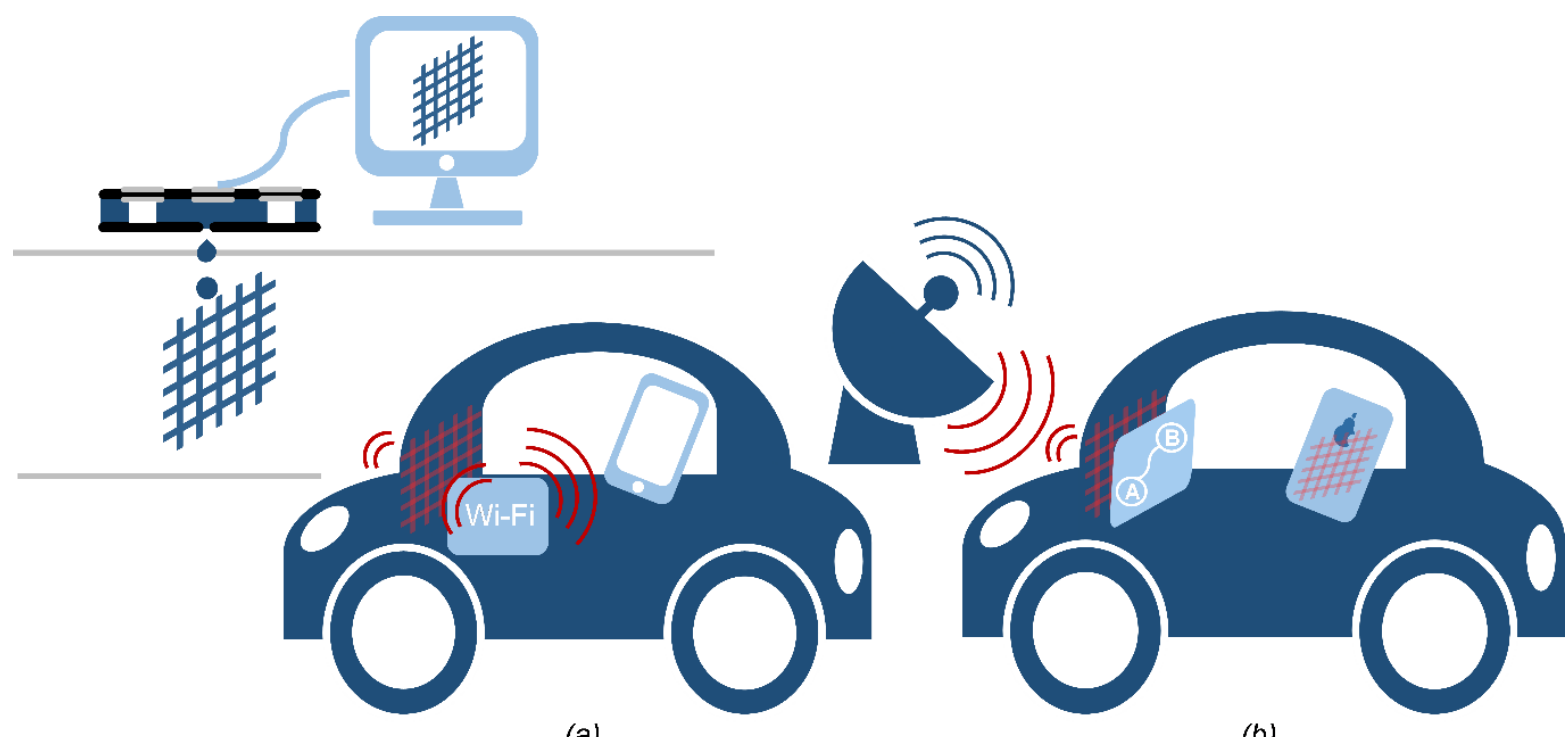

(a)

(b)

Figure 1: Manufacturing process of inkjet-printed grid and the potential application as

(a) electromagnetic wave attenuator or (b) protector of EMC sensitive devices

\section{Experimental}

\subsection{Printing Process and Materials}

The inkjet-printing process was performed with a Dimatix Materials Printer (DMP) 2831 and an installed piezoelectric printhead with 16 nozzles, which have a distance of $254 \mu \mathrm{m}$ (Figure 2). The piezoelectric elements are driven by a voltage waveform. A silver nano-particle ink PE410 (DuPont) was used and printed on a PET substrate Melinex 506 (DuPont Teijin Films) which has a thickness of $100 \mu \mathrm{m}$. The solid content of the ink is 45.2 wt\% dissolved in Butyldiglycol and octylamine [11]. The PET substrate consists of an adhesion promoting pre-treatment [12].
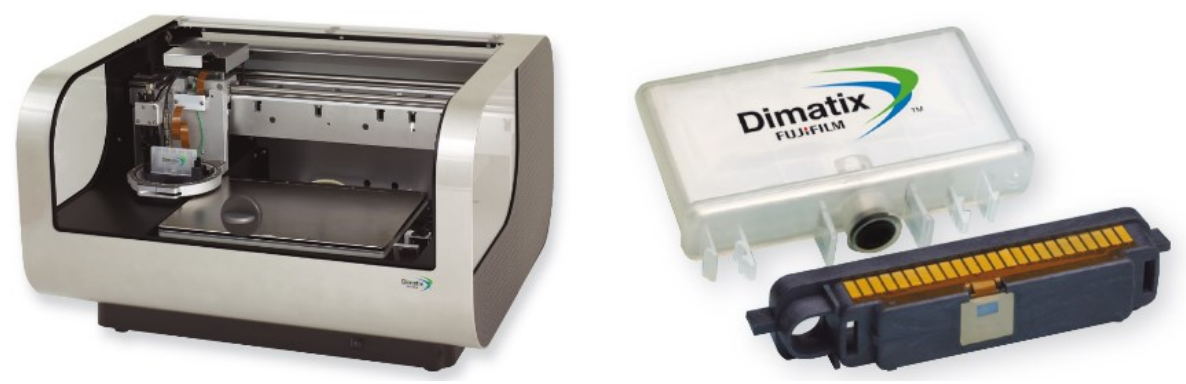

Figure 2: Dimatix Inkjet Printing System DMP 2831 and Cartridge with Printhead [13]

Preliminary experiments were implemented to evaluate the performance of the ink at different resolutions, which are defined by the drop space (DS) (distance of the deposited droplets). One pixel (px) lines with DS 15 to $45 \mu \mathrm{m}$ were printed as well as $300 \mu \mathrm{m}$ wide lines and $5 \times 5 \mathrm{~mm}^{2}$ squares with ideal DS for electrical characterization. 
The printed layers have to be sintered to evaporate the solvent, to cast out the organic non-conductive components and to encourage a grain growth. To form dense and conductive layers the samples were sintered in a convection oven for $60 \mathrm{~min}$ at $100^{\circ} \mathrm{C}$.

On the basis of the morphological and electrical evaluation of the preliminary experiments the grid pattern was simulated.

\subsection{Simulation of Pattern Design}

The grid pattern for the frequency-dependent attenuation of electromagnetic waves was simulated by the software CST Microwave Studio Suite [14]. The built up simulation design can be seen in Figure 3.

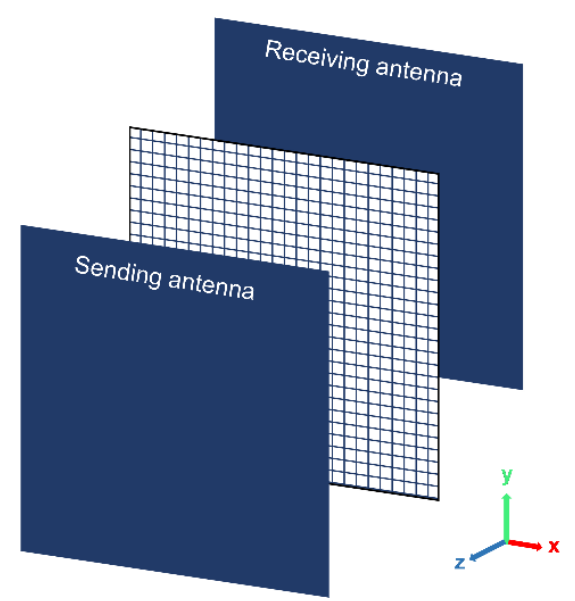

Figure 3: Simulation design - simulated grid between sending and receiving antenna

A grid pattern was chosen intentionally because of less material consumption compared to conventional full-surface coating and the aim to customize the layout design to different frequency ranges.

\subsection{Characterization}

The printed lines as well as squares were analyzed optically by microscopic images (Leica DM 4000 M), morphologically by surface profile measurement (Veeco Dektak 150) and electrically by sheet resistance measurements $\left(5 \times 5 \mathrm{~mm}^{2}\right.$ squares) by the four point Van-der-Pauw method. These measurements reveal the optimal DS to be used to reproduce the simulated pattern precisely and reliably. Open layers, blurred edges and inaccurate dimensions could affect the target design of the grid. Therefore, accurate dimensions and sharp edges are crucial.

\section{Results and Discussion}

\subsection{Preliminary Experiments}

First of all, an optical analysis of patterns printed with different DSs was implemented. As the microscopic images (Figure 4 (a)) show, the width of the lines seems to decrease by larger DSs. Since fewer droplets are deposited if a higher DS is used less material is applied onto the substrate. Due to the fact that closed, sharp and fine patterns with DS 30 to $40 \mu \mathrm{m}$ could be realized $300 \mu \mathrm{m}$ wide lines and $5 \times 5 \mathrm{~mm}^{2}$ square patterns are printed with DS 30 and $40 \mu \mathrm{m}$. These DSs seem to form close and dense layers (Figure 4 (b) and (c)).

To confirm the optical impression the line width and layer profile measurements are performed subsequently. 


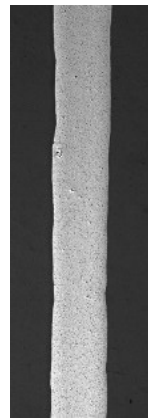

DS $15 \mu \mathrm{m}$

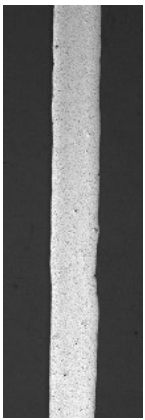

$20 \mu \mathrm{m}$

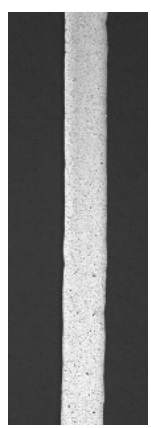

$25 \mu \mathrm{m}$

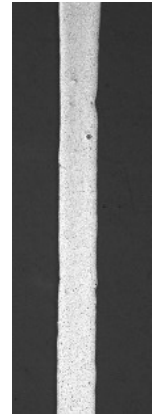

$30 \mu \mathrm{m}$

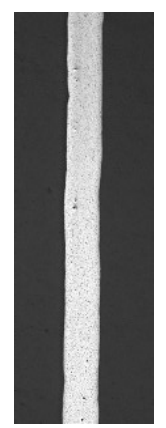

$35 \mu \mathrm{m}$

(a)

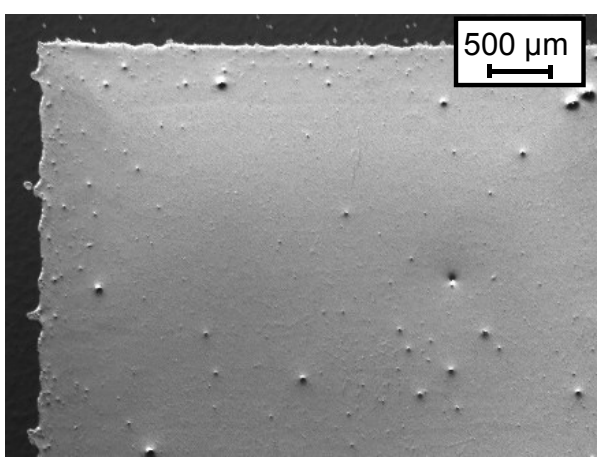

DS $30 \mu \mathrm{m}$

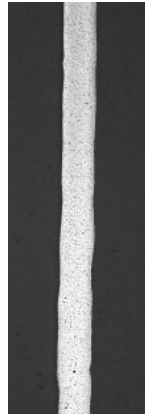

$40 \mu \mathrm{m}$

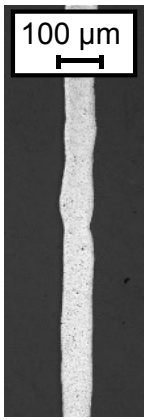

$45 \mu \mathrm{m}$

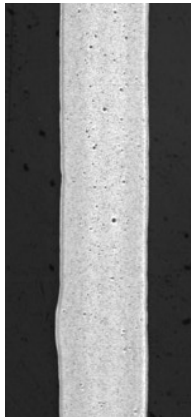

DS $30 \mu \mathrm{m}$

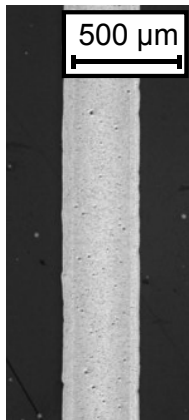

$40 \mu \mathrm{m}$

(b)

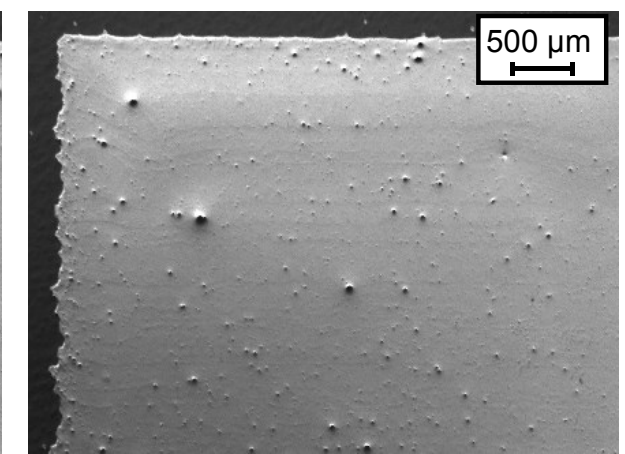

$40 \mu \mathrm{m}$

(c)

Figure 4: Microscopic images of (a) 1 px lines, (b) lines of $300 \mu \mathrm{m}$ width and (c) $5 \times 5 \mathrm{~mm}^{2}$ squares printed with different $D S$

The line width measurements (Figure 5 (a)) confirm that increasing DSs lead to reduced line widths.

For printing the $300 \mu \mathrm{m}$ wide lines DSs of 30 and $40 \mu \mathrm{m}$ were used (Figure 5 (b)). It can be noticed that the printed line widths differ from the nominal line width $(300 \mu \mathrm{m})$. This fact has to be considered in the subsequent simulation of the grid pattern.

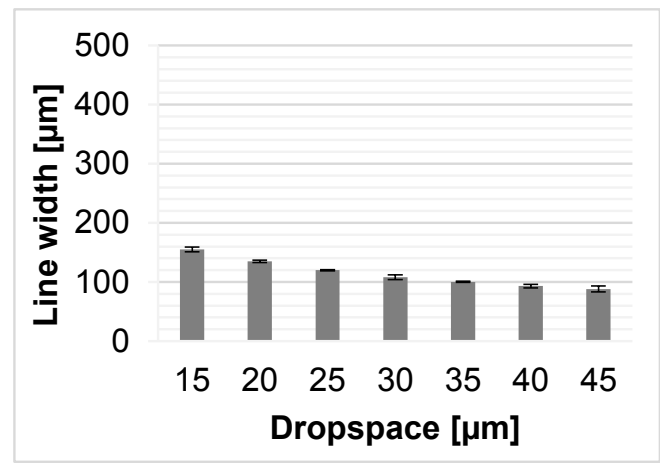

(a)

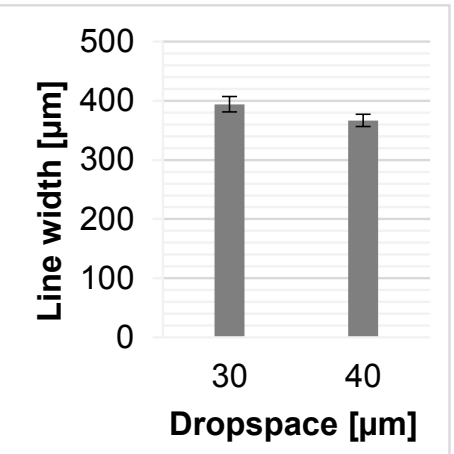

(b)

Figure 5: Average line width of (a) 1 px lines and (b) $300 \mu \mathrm{m}$ wide lines printed with different $D S$

Figure 6 demonstrates the layer morphology (a) and average layer height (b) of the printed $1 \mathrm{px}$ and $300 \mu \mathrm{m}$ wide lines. Higher DSs obviously lead to a decreased layer thickness since less material is applied onto the substrate. If $1 \mathrm{px}$ lines and $300 \mu \mathrm{m}$ wide lines are compared it can be seen that the layer height increases with broader patterns. The $1 \mathrm{px}$ lines also show a so called coffee-ring effect, this means more material is located at the edges of the lines. This effect is reduced in case of wider lines. 


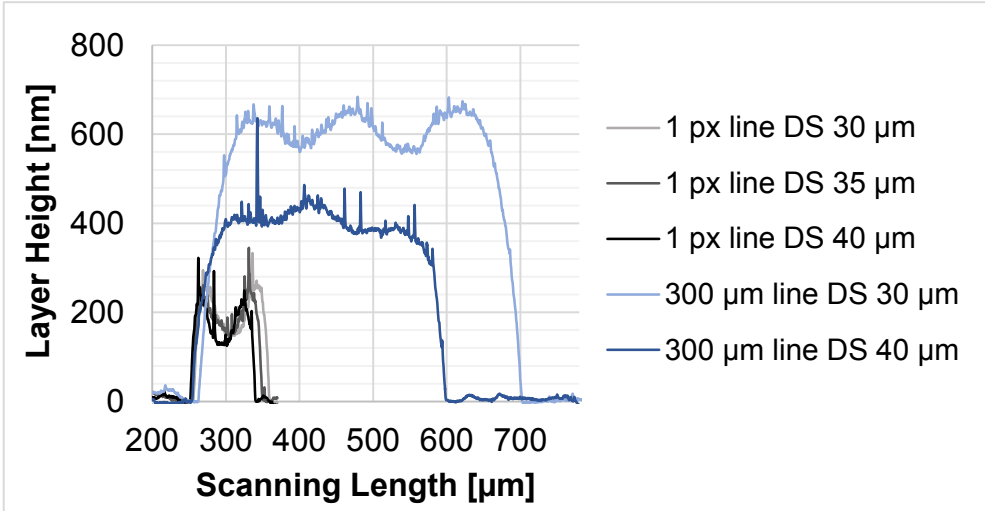

(a)

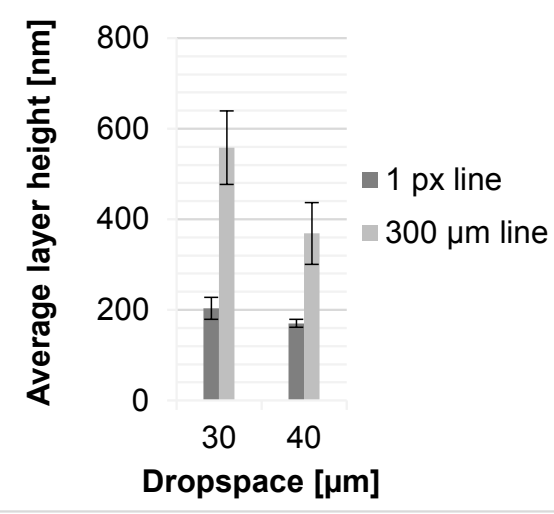

(b)

Figure 6: (a) Surface profile and (b) Average layer height of 1 px lines and $300 \mu m$ wide lines printed with different $D S$

The electrical evaluation of the preliminary printed patterns confirm the previous optical and morphological measurement results. The resistance increases by increasing DS (Figure 7) due to a smaller amount of deposited material. Nevertheless, the sheet resistance values of DS 30 and $40 \mu \mathrm{m}$ differ only slightly from each other. The electrical properties of the patterns seem sufficient for the intended simulated pattern design and the application later on.

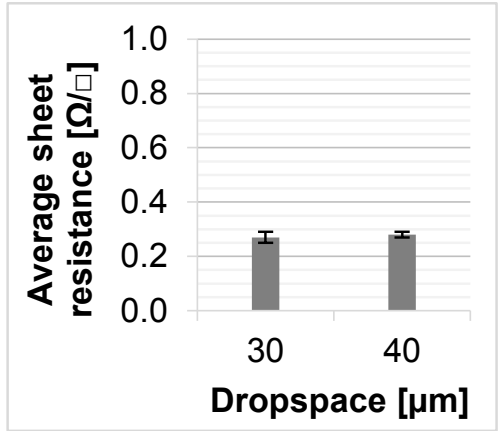

Figure 7: Average sheet resistance of patterns printed with different DS

\subsection{Simulation results}

Lines with a nominal width of $300 \mu \mathrm{m}$ and an average printed width of $367 \mu \mathrm{m}$ (DS $40 \mu \mathrm{m}$ ) respectively are considered for the simulation. The specific electrical conductivity of $9.68 \mathrm{E}+06 \mathrm{~S} / \mathrm{m}$ used for the simulation was calculated with the help of the average line thickness $(369 \mathrm{~nm})$ and the average sheet resistance $(0.28 \Omega / \square)$ of the printed layers. These values were the basis for the simulation of a grid pattern with different mesh widths at a resonance frequency between 1 and $10 \mathrm{GHz}$ (Figure 8). 


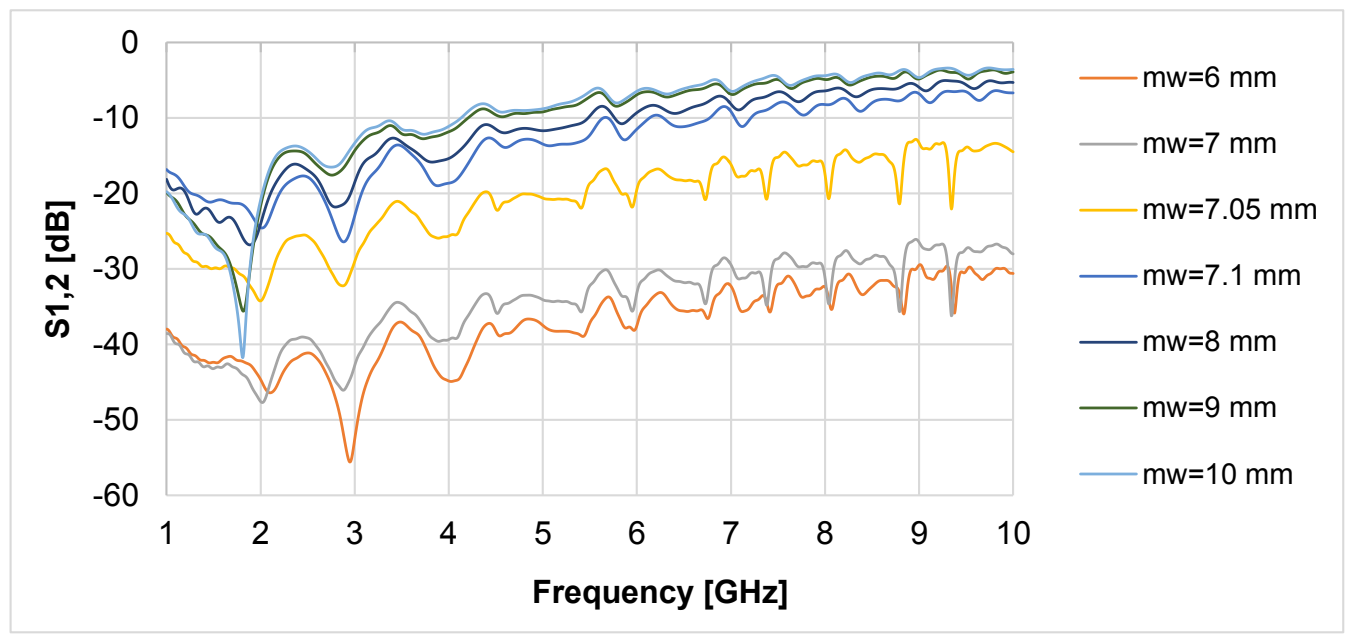

Figure 8: Frequency-dependent attenuation at different mesh widths (mw)

The simulation shown in Figure 8 demonstrates that with decreasing mesh width (mw) the attenuation effect of the resonance signal increases. There is a noticeable jump in the attenuation between mesh width $7.1 \mathrm{~mm}$ and $7.05 \mathrm{~mm}$ as well as from $7.05 \mathrm{~mm}$ to 7 . While the attenuation effects of grids with mesh widths in the range of 7.1 to $10 \mathrm{~mm}$ are only slightly different. All in all, it is demonstrated that a frequency dependent attenuation between -10 and $-50 \mathrm{~dB}$ is achievable with the given material parameters.

Additionally, the attenuation dependency on the width of the grid lines was simulated (Figure 9).

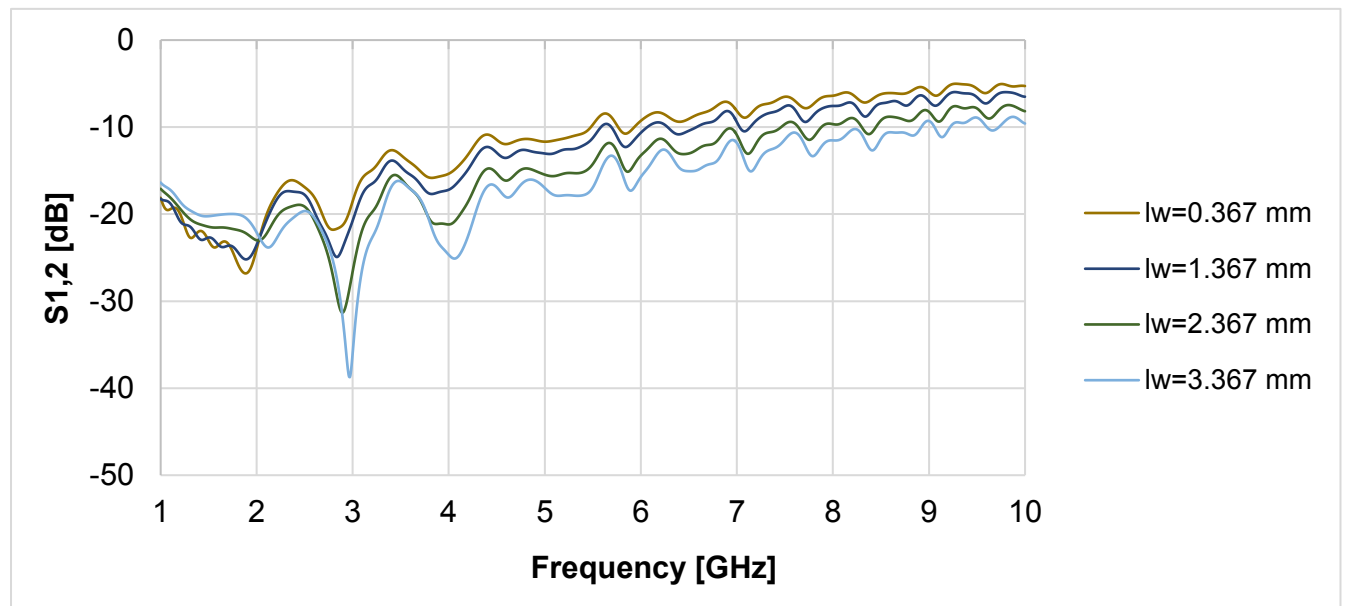

Figure 9: Frequency-dependent attenuation at different line widths (Iw), constant mesh width of $10 \mathrm{~mm}$

The simulation shown in Figure 9 demonstrates that the line width (Iw) of the grid lines has no relevant influence on the attenuation of the resonance signal. Which will be considered in further research.

All in all the simulations show that with changing of either line or mesh width of the grids an intended attenuation can be realised at various frequencies leading to various application possibilities.

\section{Conclusion}

In this research a brief characterization of a silver inkjet ink regarding line width, layer thickness and electrical performance was performed. On the basis of these fundamental experiments simulations of grid designs for the specific attenuation of electromagnetic signals were implemented. The simulation results demonstrate that the utilized silver ink has great potential to be used for grid patterns attenuating 
electromagnetic waves. The mesh design with narrow line widths as well as thin layers which can be realized especially by the inkjet printing technology lead to minimal and selective material input and therefore a resource efficient manufacturing. Simulations demonstrate that the width of the printed silver lines has less influence than the mesh width of the grid. The planned printed meshes will operate at the intended frequency of $2.45 \mathrm{GHz}$ and moreover at further frequencies. The results are the fundament for future researches where the simulated grids will be realized by the inkjet printing technology and applied for the proposed applications like local electromagnetic wave attenuation on Wi-Fi or Bluetooth transmitters and in future also for protection of electromagnetic compatibility (EMC) sensitive devices close to electromagnetic transmitters.

\section{Acknowledgements}

This work was performed within the Federal Cluster of Excellence EXC 1075 "MERGE Technologies for Multifunctional Lightweight Structures" and supported by the German Research Foundation (DFG). Financial support is gratefully acknowledged.

\section{References}

[1] Mitra, K. Y.; Hartwig, M.; Weise, D.; Baumann, R. R.: Infra-Red curing methodology for R2R manufacturing of conductive electrodes through Inkjet technology applicable for devices in the field of flexible electronics, MRS Spring 2015, San Francisco, USA, April 6-10, 2015.

[2] Zichner, R.; Sowade, E.; Baumann, R. R.: Inkjet printed WLAN antenna for an application in smartphones. Jpn. J. Appl. Phys., 53(5S3) (2014). doi: 10.7567/JJAP.53.05HB06

[3] Hartwig, M.; Gaitzsch, M.; Großmann, T. D.; Heinrich, M.; Kroll, L.; Gessner, T.; Baumann, R. R.: Investigation on an Inkjet Printed Passive Sensor for Wireless Ice Detection on Wind Rotor Blades, J. of Imag. Sci. and Tech., 60(4) (2016), pp. 40402-1-40402-7(7). doi: 10.2352/J.ImagingSci.Technol.2016.60.4.040402

[4] Belhaj, M. M.; Wei, W.; Pallecchi, E.; Mismer, C.; Roch-jeune, I.; Happy, H.: Inkjet printed flexible transmission lines for high frequency applications up to $67 \mathrm{GHz}$. In: European Microwave Week 2014: Connecting the future: IEEE, 2014, pp. 1528-1531.

[5] Sohail, S. I.: Wi-Fi transmission and multi-band shielding using single-layer frequency selective surface. In: 2016 IEEE International Symposium on Antennas and Propagation (APSURSI), June 26-July 1, 2016, Fajardo, Puerto Rico. doi: 10.1109/APS.2016.7696190

[6] Sagstetter, F.; Lukasiewycz, M.; Steinhorst, S.; Wolf, M.; Bouard, A.; Harris, W. R.; Jha, S.; Peyrin, T.; Poschmann, A.; Chakraborty, S.: Security Challenges in Automotive Hardware/Software Architecture Design. in Design, Automation \& Test in Europe Conference \& Exhibition, March 18 - 22, 2013, Grenoble, France; proceedings, Piscataway, NJ: IEEE, 2013, pp. 458-463.

[7] Weng, W.-C.; Ye, H.-C.: Electromagnetic compatibility designs for electronic circuits in electric vehicles. In: 2016 IEEE 5th Asia-Pacific Conference on Antennas and Propagation (APCAP), Kaohsiung, Taiwan, July 26-29, 2016. doi: 10.1109/APCAP.2016.7843180

[8] Syed, I. S.; Ranga, Y.; Matekovits, L.; Esselle, K. P.; Hay, S. G.: A Single-Layer FrequencySelective Surface for Ultrawideband Electromagnetic Shielding. IEEE Trans. on Electromagn. Compat., 56(6) (2014). doi: 10.1109/TEMC.2014.2316288

[9] Drakakis, E.; Kymakis, E.; Tzagkarakis, G.; Louloudakis, D.; Katharakis, M.; Kenanakis, G.; Suchea, M.; Tudose, V.; Koudoumas, E.: A study of the electromagnetic shielding mechanisms in the $\mathrm{GHz}$ frequency range of graphene based composite layers. Appl. Surf. Sci., 398, pp. 15-18, 2017. doi: 10.1016/j.apsusc.2016.12.030

[10] Wang, L.-L.; Tay, B.-K.; See, K.-Y.; Sun, Z.; Tan, L.-K.; Lua, D.; Electromagnetic interference shielding effectiveness of carbon-based materials prepared by screen printing. Carbon, 47(8), pp. 1905-1910, 2009. doi: 10.1016/j.carbon.2009.03.033

[11] http://www.dupont.com/content/dam/dupont/products-and-services/electronic-and-electricalmaterials/documents/prodlib/PE410-Data-Sheet.pdf [last access: June 21, 2017].

[12] http://www.micel.fr/IMG/pdf/Melinex-506 FT micel-4.pdf [last access: June 21, 2017]. 
Technologies for Lightweight Structures 1(2) (2017)

[13] https://www.fujifilmusa.com/shared/bin/DMP-2831 Datasheet.pdf [last access: June 21, 2017].

[14] http://www.cst.com/Content/Products/MWS/Overview.aspx [last access: August 14, 2017]. 